\title{
edoc
}

Institutional Repository of the University of Basel

University Library

Schoenbeinstrasse 18-20

CH-4056 Basel, Switzerland

http://edoc.unibas.ch/

Year: 2004

\section{Vom Zwiespalt des Historischen}

\author{
Angehrn, Emil
}

Posted at edoc, University of Basel

Official URL: http://edoc.unibas.ch/dok/A5251798

Originally published as:

Angehrn, Emil. (2004) Vom Zwiespalt des Historischen. In: Généalogie de la pensée moderne : volume d'hommages à Ingeborg Schüssler = Genealogie des neuzeitlichen Denkens : Festschrift für Ingeborg Schüssler. Frankfurt am Main, S. 365-380.

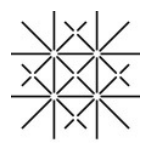




\title{
VOM ZWIESPALT DES HISTORISCHEN
}

\author{
Emil Angehrn
}

\section{Ambivalenzen des Geschichtsbewusstseins in der Moderne}

Wozu Geschichte? Welches Interesse sollen wir an der Geschichte nehmen? Wie sollen wir mit Geschichte umgehen? Auf solche Fragen haben die Menschen im Laufe der Geschichte die vielfältigsten Antworten gegeben. Nicht nur sind die Formen der Geschichtsschreibung, die Regeln der historischen Erzählung, Erklärung und Interpretation in verschiedenen Zeiten und Kulturen ganz unterschiedlich bestimmt worden. Auch auf die grundlegende Frage, ob und wieviel Geschichte wir brauchen, finden wir die unterschiedlichsten Antworten. Nicht für alle Epochen und nicht für alle Gesellschaften ist historisches Bewusstsein ein wichtiges Kulturgut. Indessen geht es hier nicht nur um ein Schwanken in der Einschätzung historischer Kultur im Vergleich der Epochen. Vielmehr geht es um einen grundlegenden Zwiespalt des Historischen, der sich im Durchgang durch die Zeiten, aber ebenso in einer heutigen Reflexion manifestiert. Es ist ein Zwiespalt, der selber einen historischen Ort hat. Zumindest scheint es plausibel, ihn als Kennzeichen des Geschichtsdenkens der Moderne, nach dem Zusammenbruch sowohl der großen Geschichtsphilosophie wie des klassischen Historismus zu sehen.

Diesem Zwiespalt möchte ich in den folgenden Ausführungen nachgehen. Ich gehe dabei von Nietzsches bekannter Diagnose der historischen Kultur in der Zweiten Unzeitgemäßen Betrachtung: Vom Nutzen und Nachtheil der Historie für das Leben aus und versuche, diese Diagnose als Hintergrund für eine Diskussion neuerer Konzepte - vor allem von Michel Foucault und Jacques Derrida - zu verwenden. Es scheint mir durchaus bemerkenswert, in welcher Prägnanz der junge Nietzsche Aspekte und Linien der Auseinandersetzung um 
Geschichte benennt, die ein Jahrhundert später, in verändertem Kontext, nichts von ihrer Aktualität verloren haben.

Ich will die Hauptlinien des Gedankens vorweg in folgender Weise umreißen. Der Zwiespalt in unserem Umgang mit Geschichte ist selbst ein zweifacher. Zum einen ist es die grundlegende Ambivalenz des historischen Bewusstseins, d.h. die Frage, $a b$ wir überhaupt ein Interesse an historischer Erinnerung nehmen (sollen) oder ob wir eher Geschichte verdrängen, uns von ihr befreien, uns gegen die Last der Vergangenheit zur Wehr setzen (sollen). Auf der anderen Seite ist es die Frage, in welcher Weise wir uns auf Geschichte beziehen (sollen), welches die richtige - wahrheits- und wissenschaftsfähige, authentische, humane - Weise ist, Geschichte zu rezipieren, kritisch zu beurteilen und anzueignen. Es sind zwei Fragerichtungen, die ineinander verflochten sind, die aber auch unabhängig voneinander ihre Schärfe und Bestimmtheit haben.

Bei Nietzsche treten sie uns in zwei distinkten Figuren entgegen: zum einen in der Opposition zwischen dem Historischen auf der einen Seite, dem Unhistorischen und Überhistorischen auf der anderen Seite; zum anderen in der Unterscheidung dreier Arten von Historie, die unter je spezifischen Hinsichten als lebensförderlich oder lebensfeindlich erkannt, befürwortet oder verurteilt werden. Klärungsbedürftig bleibt, wie sich die grundlegende Auseinandersetzung um das Historische mit der differenzierenden Analyse der unterschiedlichen Typen der Historie verschränkt (und wieweit diese sich zu einer gemeinsamen Geschichtskultur verbinden oder als heterogene Orientierungen bestehen bleiben).

Beide Fragestellungen sind ebenso in neueren Diskussionen - sowohl im theoretischen Disput wie in der öffentlichen Auseinandersetzung auszumachen, teils in zugespitzter Weise ausformuliert. Die zweite Frage, die Frage nach den unterschiedlichen Modi und der richtigen Gestalt des historischen Bewusstseins, bildet einen Leitfaden der Methodendiskussionen in den Geschichts- und Kulturwissenschaften der letzten zwei Jahrhunderte. Ebenso ist die erste Frage, die Frage nach dem grundsätzlichen Interesse an Geschichte, eine sowohl in der kulturellen Öffentlichkeit wie im Fachdisput kontrovers behandelte Frage. Die aktuelle Konjunktur des Themas « Gedächtnis und Erinnerung » bei Tagungen und in Fachpublikationen, anlässlich der Gründung von Museen und Gedenkstätten, in historischen Fernsehsendungen und öffentlichen Diskussionen ist Indiz eines Bewusstseinszustandes, dem ein bestimmtes Verhältnis bzw. Nichtverhältnis zur Geschichte zum offenen Problem geworden ist. Doch nicht nur im moralisch-politisch besetzten Feld der 
Aufarbeitung der Realgeschichte, auch im Binnenbereich kultureller Tradition ist die Frage, ob und wozu wir Geschichte brauchen, alles andere als einvernehmlich beantwortet. Um nur als Beispiel die Philosophie selbst zu nennen: Auch wenn es zur gängigen Praxis des Philosophierens gehört, Sachthemen im Dialog mit historischen Positionen $\mathrm{zu}$ erörtern, ist die prinzipielle Frage, ob und wie sich Philosophie mit ihrer Geschichte auseinanderzusetzen und diese Auseinandersetzung in ihre systematische Arbeit $\mathrm{zu}$ integrieren habe, weithin kontrovers und offen. Analoges gilt für andere Disziplinen und weite Bereiche des kulturellen Lebens.

\section{Nietzsches zweifacher Einspruch gegen die historische Kultur}

Zum besonderen Profil von Nietzsches Abrechnung mit der herrschenden Geschichtskultur gehört, wie gesagt, der zweifache Ansatz der Kritik. Zum einen setzt er sich mit Historie überhaupt, zum anderen mit drei spezifischen Ausprägungen des Historischen auseinander.

Nach der ersten Hinsicht präsentiert uns Nietzsche eine der dezidiertesten Gegenwendungen zur historischen Kultur. Sie markiert nicht Geschichtsabstinenz oder -indifferenz, sondern eine Gegnerschaft, die nur aus der Konfrontation mit einem blühenden, ja wuchernden (in anderer Hinsicht leblos-abstrakten) Geschichtsbetrieb zu verstehen ist. Nicht nur wird wie im 19. Jahrhundert bei vielen Autoren - von Ranke über Marx bis Burckhardt - die klassische Geschichtsphilosophie als unzulässige Hypostasierung zurückgewiesen. Weit darüber hinaus geht es darum, eine bestimmte Ausprägung historischer Kultur, teils Grundlagen des geschichtlichen Lebens selbst, in Frage zu stellen. Zwar ist Nietzsches Text äußerlich von einer Symmetrie des Nutzens und Nachteils der Historie, einer Komplementarität von Leben und Geschichte, durchzogen; " das Unhistorische und das Historische ", sagt er, « ist gleichermaßen für die Gesundheit eines Einzelnen, eines Volkes und einer Kultur nötig » (252) ${ }^{1}$. Dennoch zielt die Abhandlung nicht einfach darauf, beide Potenzen zum Ausgleich zu bringen. Sie setzt durchaus einseitig an, indem sie vorrangig die Lebensfeindlichkeit der Historie herausarbeitet, und sie behält diese Einseitigkeit in gewissem Maße bei, indem sie das Unhistorische im Ganzen als das « wichtigere und ursprünglichere » Element präsentiert (251).

\footnotetext{
${ }^{1}$ Friedrich Nietzsche, Unzeitgemässe Betrachtungen. Zweites Stück: Vom Nutzen und Nachtheil der Historie für das Leben, KSA 1, S. 243-334, hier S. 252. Die folgenden Zitatnachweise im Text beziehen sich auf diese Schrift.
} 
Die Abhandlung bleibt stärker an der Frage ausgerichtet, bis zu welchem Grad das Leben Geschichte erträgt, als daran, bis zu welchem Grad es sie « überhaupt brauche " (257); sie beleuchtet primär die Voraussetzungen einer lebensgerechten Historie, nicht deren Interesse an ihm selbst. Geschichte kommt stärker in ihrer auflösend-destruktiven, Leben in seiner affirmativ-konstruktiven Potenz in den Blick. $\mathrm{Zu}$ fragen ist nach den Kriterien der ungleichen Akzentuierung. In dieser einseitigen Frontstellung stellt sich Nietzsche gegen den Geist der Zeit, ist seine Schrift im pointierten Sinn unzeitgemäß - auch wenn sie sich gerade darin, sowohl diagnostisch wie therapeutisch (im Wirken " gegen die Zeit und dadurch auf die Zeit und hoffentlich zu Gunsten einer kommenden Zeit » [247]), als das eigentlich Zeitgemäße versteht.

Den entscheidenden Punkt nennt Nietzsche in einem Satz, den Walter Benjamin seiner zwölften geschichtsphilosophischen These vorangestellt hat: « Wir brauchen die Historie, aber wir brauchen sie anders, als sie der verwöhnte Müssiggänger im Garten des Wissens braucht » (245). Historie, wo sie affirmativ bejaht wird, steht im Dienst der Tat und des Lebens: « Nur soweit die Historie dem Leben dient, wollen wir ihr dienen » (245); im Übermaß gepflegt, wird sie lebensfeindlich und destruktiv. Verschiedene Motive gehen in Nietzsches Beschreibung des Antagonismus von Historie und Leben ein. Das eine ist die Lebens- und Gestaltungskraft, die durch das Offensein der Horizonte, das Außersichsein der orientierungslosen Gelehrsamkeit gelähmt wird. Mit einer mehrfach wiederkehrenden Metapher beschreibt Nietzsche das Abblenden dieser offenen Horizonte als schützende « Atmosphäre [...], in der sich Leben allein erzeugt, um mit der Vernichtung dieser Atmosphäre wieder zu verschwinden » (252). Zwar wird damit nicht der Immediatismus des Lebens verabsolutiert: Das Unhistorische ist das Fundament, auf dem etwas Gesundes wachsen soll, aber als Fundament nicht das Ganze. Erst durch die bewusste Kraft, " das Vergangene zum Leben zu gebrauchen und aus dem Geschehen wieder Geschichte zu machen, wird der Mensch zum Menschen »(253). Dennoch sieht Nietzsche die plastische Gestaltungskraft nicht vorrangig im produktiven Umgang mit Geschichte, in historischer Sinnkonstruktion oder in der Bildung historischer Identität, sondern im Widerstand gegen die Vergangenheit am Werk. Dies hängt damit zusammen, dass Geschichte zunächst ein Sichverlieren ist.

In zugespitzter Form schildert Nietzsche dieses Verhältnis in der Dialektik von Erinnern und Vergessen. In direkter Umkehrung traditioneller Geschichtsanschauung, welche an die Kraft der Erinnerung gegen die natürliche Vergessenstendenz appelliert, meint Nietzsche, gegen das Verhaftetsein im 
Gedächtnis die « Kraft zu vergessen » (250) mobilisieren zu müssen. Als Leitidee fungiert die quasi-ontologische, auch ästhetische Vorstellung eines vollen Einsseins-mit-sich, einer Selbstpräsenz und Selbstaffirmation - gegen Selbstzerstreuung, Unvollendetsein, Aufgehen im Anderen. Nicht das Vergessen ist hier Selbstflucht; vielmehr ist die Erinnerung ein Verhaftetsein im Gewesenen, das nie zur vollen Deckung mit sich kommt, " ein nie zu vollendendes Imperfectum » - nicht weil sie immer unvollständig wäre, sondern weil sie sich ans Fremde ausliefert, ein Sich-selbst-Verneinen ist (249). Historische Reflexion lässt das Jetzt im Horizont von anderem erscheinen, sie virtualisiert, vergleicht, überdenkt, stellt in Frage. Die in sich erfüllte, plastische Selbstpräsenz bedingt eine Geschlossenheit, die sich im Historischen verflüchtigt und die der « Kraft » des Vergessens bedarf.

Doch nicht nur das Leben - das Unhistorische - steht im Gegensatz zur Geschichte. Ernst zu nehmen ist auch das andere Gegengift, das Nietzsche gegen die historische Krankheit verschreibt: das Überhistorische. Nicht hinter die Geschichte zurück-, sondern über sie hinauszugehen ist die wahre Befreiung vom Historischen: In den " höchsten Exemplaren » des Menschlichen (317), in denen Nietzsche den Wert der Geschichte - gegen die Fokussierung auf die Entwicklung und das Ziel - erkennt und als deren Repräsentanten er in der Dritten Unzeitgemäßen die «Philosophen, Künstler und Heiligen » sieht (380), ist nicht nur die Kraft des Lebens, sondern auch etwas von dieser Geschichtstranszendenz realisiert. Allerdings verbleibt auch hier eine Ambivalenz, wenn Nietzsche nach der Empfehlung jener beiden Gegengifte zur Historie abschließend feststellt, dass, zumal " wir, die Historisch-Kranken », wahrscheinlich « auch an den Gegenmitteln zu leiden haben » (331). Im Ganzen umreißt Nietzsche eine entschieden geschichtsskeptische Position, die aber davor zurückschreckt, zur Gänze auf die Seite des Unhistorisch-Lebendigen oder des Überhistorischen zu setzen, wohl wissend, dass auch darin das menschliche Leben sich nur partiell vollenden kann, ja, in Wahrheit in eminenter Weise gefährdet bleibt.

$\mathrm{Zu}$ Nietzsches Einschätzung des Historischen gehört nun nicht nur das Schwanken in der umfassenden Wertung der Erinnerung, sondern die interne Differenzierung in der Wirkungsweise des Historischen. Die Titelfrage nach Nutzen und Nachteil der Historie beantwortet er nicht generell, sondern in direkter Spezifizierung für drei typische Ausprägungen historischer Kultur, denen er unterschiedliche Bedürfnisse des Lebendigen zuordnet: Als monumentalische gehört die Historie dem Lebendigen als « dem Tätigen und Strebenden ", als antiquarische " dem Bewahrenden und Verehrenden », als 
kritische "dem Leidenden und der Befreiung Bedürftigen » (258). Das Bemerkenswerte an Nietzsches Schilderung dieser drei Typen, die hier nicht im Einzelnen zu erörtern sind, liegt zum einen darin, dass er drei überaus prägnante, gleichsam idealtypische Kristallisationen historischen Bewusstseins zeichnet, die wir in vielen ideen- und kulturgeschichtlichen Konstellationen wiedererkennen können und die auch für eine Analyse heutiger Geschichtskultur diagnostische Kraft haben. Sie definieren drei unterschiedliche Stoßrichtungen, nach denen menschliches Leben sich im Geschichtsbezug vollzieht: Indem es im Gedächtnis des Großen und Mächtigen eine Stütze des eigenen Schaffens findet, indem es in vergangenheitszugewandter Pietät in seiner Geschichte heimisch wird, und indem es die Vormacht des Vergangenen bricht, sich kritisch mit der Geschichte auseinandersetzt, sich gewissermaßen von ihr befreit. Je nach Kontext und eigener Disposition ist die eine oder andere Art des Umgehens mit Geschichte verlangt; jede für sich artikuliert einen wesentlichen Zug des Historischen und kann ein vitales Interesse des historischen Bewusstseins befriedigen. Zum anderen gehört zur Sachhaltigkeit von Nietzsches Erörterung, dass sie jede dieser Formen in ihrer Ambivalenz, ihrer Lebensförderlichkeit und Lebensfeindlichkeit vergegenwärtigt - ohne dass beide Seiten sich einfach aufhöben oder irgendwie in der Mitte zum Ausgleich kämen. Die von Nietzsche betonten Gefahren sind zum Teil die direkte Kehrseite der genannten affirmativen Potenzen des Historischen: Gefahren der Verfälschung und Verzeichnung, der blinden Verehrung des Faktischen, der Passivität, des Verlusts des Maßes, des abstrakten Kosmopolitismus, der Ironie und der Entwurzelung. Unter zahlreichen Facetten stellt Nietzsche vor Augen, wie das historische Bewusstsein zur Fessel und zur Gegenkraft des erneuerndgestaltenden Lebens werden kann. Vorrangig bleibt auch hier die Frage, unter welchen Voraussetzungen der Mensch Geschichte deuten kann und darf; wieweit er umgekehrt nur aus der Geschichte die Kraft zum Erbauen der Zukunft hat, bleibt unterbestimmt.

Das Intrikate der von Nietzsche vorgestellten Sicht des Historischen besteht offensichtlich darin, dass nicht auf der Hand liegt, wie die unterschiedlichen Stoßrichtungen in Ausgleich zu bringen, ja, in welcher Weise sie überhaupt zueinander in ein Verhältnis zu setzen sind. Dies betrifft zum einen innerhalb der einzelnen Formen der Historie ihre lebensbejahenden und destruktiven Potenzen, zum anderen das gegenseitige Verhältnis dieser heterogenen, teils direkt antagonistischen Stoßrichtungen der Geschichte, schließlich das übergreifende Verhältnis zwischen dem unhistorischen Gestaltungsdrang des Lebendigen, der Kulturleistung historischer Konstruktion und dem 
überhistorischen Transzendieren des Wandelbar-Zeitlichen. Die Konstellation dieser heterogenen Motive bildet die exemplarische Signatur einer in sich zwiespältigen Geschichtlichkeit menschlichen Lebens.

\section{Foucault: Historie zwischen Archäologie und Genealogie}

In paradigmatischer Weise hat Michel Foucault ein verändertes Modell historischer Forschung entwickelt, das nicht zuletzt wesentliche Impulse in Nietzsches historischer Praxis und Reflexion findet. Foucaults Werk ist im Wesentlichen als historische Forschung durchgeführt, wie schon die Titel seiner Bücher belegen: Histoire de la folie à l'âge classique (1972), La naissance de la clinique. Une archéologie du regard médical (1963), Les mots et les choses. Une archéologie des sciences humaines (1966), L'archéologie du savoir (1969), Surveiller et punir. La naissance de la prison (1975), Histoire de la sexualité (1976 ff.). Fast obsessiv kreisen die Werke um die Brennpunkte Geburt, Geschichte, Archäologie. Im folgenden sollen nicht diese materialen Studien im Zentrum stehen, sondern drei kurze programmatische Texte, die chronologisch in der Mitte zwischen den frühen und den späteren Werken liegen: die Einleitung zur Schrift Archäologie des Wissens (fr. 1969), die Antrittsvorlesung am Collège de France Die Ordnung des Diskurses (gehalten 1970, publ. fr. 1971) und der Aufsatz Nietzsche, die Genealogie, die Historie (fr. 1971). ${ }^{2}$

Unter verschiedenen Hinsichten charakterisiert Foucault die von ihm postulierte Historie als Umkehrung des traditionellen Verständnisses der Geschichte. Eine erste Umkehrfigur lässt sich anhand des Leitbegriffs der Archäologie charakterisieren. Archäologie ist die "Disziplin der stummen Monumente, der bewegungslosen Spuren, der kontextlosen Gegenstände und der von der Vergangenheit hinterlassenen Dinge $»^{3}$. Es ist, mit anderen Worten, die Welt der materiellen, äußerlichen, zerstreuten, nicht in ihrem Zusammenhang und ihrem Bedeutungsgehalt gegebenen Dinge, die wir durch Kontextualisierung, Entzifferung und Interpretation historisch bearbeiten und lesbar, zum Bestandteil einer Geschichte machen. Gehört es zur Aufgabe der Historie nach traditionellem Verständnis, die «Monumente der Vergangenheit

\footnotetext{
${ }^{2}$ Michel Foucault, L'archéologie du savoir, Paris, Gallimard, 1969, S. 10-28; L'ordre du discours, Paris, Gallimard, 1971 ; "Nietzsche, la généalogie, l'histoire ", in: Daniel DefERT/François Ewald (Hg.), Dits et écrits 1954-1988. Bd. II: 1970-1975, Paris, Gallimard, 1994, S. 136-156.

${ }^{3}$ Foucault, L'archéologie du savoir, Paris, Gallimard, 1969, S. 15 (dt.: Archäologie des Wissens, Frankfurt/Main, Suhrkamp, 1973, S. 15).
} 
[...] in Dokumente zu transformieren ", d.h. in etwas, das üblicherweise als Zeugnis einer Sinnbekundung gilt, und damit die Spuren des Vergangenen gleichsam wie Äußerungen - statt als nicht-intentionale Residuen und Abdrücke - zu lesen, so verfolgt die von Foucault praktizierte historische Forschung den umgekehrten Weg, « die Dokumente in Monumente » zu verwandeln und die Spuren, statt sie zu entziffern, in ihre Elemente zu zerlegen. Von solcher Geschichte könnte man sagen, dass sie « zur Archäologie tendiert - zur immanenten Beschreibung des Monuments $»^{4}$.

Mit dieser Wendung zur Äußerlichkeit und Materialität verbinden sich andere Umkehrungen, welche die Logik des Historischen betreffen. Ziel der Historie ist nicht die einheitliche Sinnkonstruktion, sondern die Sinnauflösung, nicht die zusammenfassende Integration des Vielen, sondern das archivierende Festhalten des Heterogenen, nicht die verinnerlichende Zentrierung einer geschichtlichen Konstellation auf ihren Sinn hin, sondern das veräußerlichende, materialisierend-verräumlichende Auseinanderlegen in die Elemente und deren Beziehungen. Bezeichnend sind die Titel, unter die Gilles Deleuze seine Besprechung zweier Bücher von Foucault gestellt hat: "Der neue Archivar » und « Ein neuer Kartograph $»^{5}$. Archiv und Kartographie, sonst Instrumente und Hilfsdisziplinen der Geschichtsschreibung, werden $\mathrm{zu}$ deren Modell. Strukturelle und normative Leitbegriffe der historischen und geschichtsphilosophischen Formgebung werden in ihr Gegenteil gekehrt: Statt des Ausgriffs aufs Ganze bestimmt der mikrologische Blick die Historie, statt der synthetisierenden Gestaltung das analysierende Sezieren, statt der kontinuierlichen Verflechtung das Insistieren auf Brüchen, statt der Zweckmäßigkeit des Geschehens das mechanische Spiel der Kräfte, statt der Lenkung durch den Geist das Einschreiben in den Leib. Ging es Hegels Geschichtsphilosophie darum, die Vernunft im Geschehen auszumachen und den Zufall zu beseitigen, ist Historie hier einem "Spiel der Geschichte " zugewandt, das ganz dem "Zufall des Kampfes » und «Würfelspiel des Ereignisses » erwächst. ${ }^{6}$ Ganz dem Material, dem archäologischen Befund sich zuzuwenden, ohne teleologisch-rationalistische Überformung, definiert einen « positivistischen » Historismus, den Foucault ohne Nostalgie nach einer identitätsstiftenden und legitimierenden Geschichte übernehmen möchte und

\footnotetext{
${ }^{4}$ Foucault, L'archéologie du savoir, S. 14f. (dt. S. 15).

${ }^{5}$ Critique 274, 1970; 343, 1975 (dt. in: Gilles Deleuze/Michel Foucault, Der Faden ist gerissen, Berlin, Merve, 1977).

${ }^{6}$ Michel FoucaulT, « Nietzsche, la généalogie, l'histoire », S. 145, 147 (dt.: « Nietzsche, die Genealogie, die Historie ", S. 95, 98).
} 
den er in diesem Sinn als einen «glücklichen Positivismus » versteht und empfiehlt. $^{7}$

Terminologisch stellt Foucault ein solches historisches Vorgehen unter den Titel der Genealogie - in Anlehnung an Nietzsches Genealogie der Moral, deren Ansatz er als transformierende Radikalisierung der Kritik des Historischen in der Zweiten Unzeitgemäßen Betrachtung liest. Der Begriff des Genealogischen gibt zu verstehen, dass es nicht nur um die archivalisch-archäologische Sammlung, sondern durchaus um die Rekonstruktion einer Herkunft und eines Werdens geht, die sich aber vom Ansatz klassischer Geschichtskonstruktion grundlegend unterscheidet. Ein zentraler Differenzpunkt ist etwa die Vorstellung des Ursprungs. Bleibt traditionelles Geschichtsdenken dem metaphysischen Ursprungsdenken verhaftet, welches im Ersten und Anfänglichen das Wesen und den Grund sieht, so geht es der Genealogie darum, die kontingenten, unzusammenhängenden, niedrigen, durch Herrschaft und Gewalt bestimmten Fakten nachzuzeichnen, aus denen etwas hervorgegangen ist. Gegen einen affirmativen Grund, der dem aus ihm Entspringenden Würde, Sicherheit und Legitimität verleiht, vollzieht solche Nachzeichnung eine « boshafte Begründung [...], die ihre Idole zerbricht » $(\text { Deleuze })^{8}$ und die Legitimation widerruft. Vollends verleiht sie dem aus dem Ursprung Kommenden nicht die feste Identität, die ein metaphysisches Ursprungsdenken unterstellt. Vielmehr läuft die genealogische Analyse darauf hinaus, dasjenige, das sie rekonstruiert, zugleich zu zersetzen, es gewissermaßen im Medium seiner Auflösung zu konstituieren. In Anlehnung an die drei Typen der Historie in Nietzsches Frühschrift sieht Foucault im Konzept der Genealogie drei Arten einer zersetzenden Historie angelegt, eine wirklichkeitszersetzende, identitätszersetzende und wahrheitszersetzende historische Praxis: wirklichkeitszersetzend als Parodie, die dem europäischen Mischmenschen, " der nicht weiß, wer er ist und welchen Namen er zu tragen hat " ${ }^{9}$, in Verkehrung der monumentalischen Verehrung aus der Kostümkammer der Geschichte Masken und Ersatzidentitäten anbietet, identitätszersetzend als

\footnotetext{
${ }^{7}$ Foucault, L'ordre du discours, S. 72 (dt. Die Ordnung des Diskurses, München, Carl Hanser, 1974, S. 48); in der Archäologie des Wissens spricht er analog von einem « fröhlichen » Positivismus (S. 182).

${ }^{8}$ Gilles DELEUZE, « L'homme, une existence douteuse », in: Le Nouvel Observateur, 1.6.1966 (dt. « Der Mensch, eine zweifelhafte Existenz », in: Gilles Deleuze /Michel Foucault, Der Faden ist gerissen, a.a.O., S. 13-20, hier S. 16).

${ }^{9}$ Michel Foucault, « Nietzsche, la généalogie, I'histoire », S. 153 (dt. « Nietzsche, die Genealogie, die Historie », S. 104).
} 
Auflösung des antiquarischen Gedächtnisses, welche « die Wurzeln unserer Identität $[\ldots]$ in alle Winde zerstreut ${ }^{10}$, wahrheitszersetzend, sofern sie sich - in radikalisierender Weiterführung der kritischen Verurteilung des Vergangenen - gegen die Historie als reine Erkenntnis wendet und sich vom Willen zum Wissen und zur Wahrheit befreit.

In seiner dominierenden Stoßrichtung könnte man das anvisierte Geschichtsverhältnis durch die Verbindung eines kritischen mit einem genealogischen Impuls definieren. ${ }^{11}$ Es geht darum, etwas in seiner Entstehung und seinem Werden zu erfassen und dabei zugleich das verfestigte Selbstbild und die Oberflächengestalt, in welcher es erscheint, zu zerschlagen. Historische Wahrnehmung ist immer auch Selbstkorrektur, Wahrnehmung von außen und vom Anderen her. Indessen ist es bei allem Nachdruck der Kritik wichtig, die Leistung der Historie nicht im Effekt des Auflösens und Zersetzens aufgehen zu lassen. Die weit ausgreifenden geschichtlichen Untersuchungen, die gerade Foucault vorlegt, erschöpfen sich in keiner Weise darin, ein falsches, einseitiges Bild des Wahnsinns, der Humanwissenschaften, des Strafwesens oder der Sexualität zu destruieren; der Impuls einer kritisch-genealogischen Historie dient ebenso einer bestimmten Formierung von Sinngestalten, in deren Medium wir uns über uns und die Welt verständigen. Auch die in dreifachem Sinn zersetzende Historie dient einer Neubeschreibung der Welt und unserer selbst. So bleibt auch hier ein Spannungsverhältnis sowohl zwischen unterschiedlichen Stoßrichtungen des historischen Bewusstseins wie zwischen dem Abwerfen der Geschichte und ihrer Aneignung. In einer nochmals veränderten Konstellation begegnet uns diese übergreifende Ambivalenz und innere Heterogenität des Historischen bei Jacques Derrida.

\section{Derrida: Dekonstruktion und historischer Sinn}

Es könnte auf den ersten Blick abwegig erscheinen, die Dekonstruktion im Kontext des historischen Bewusstseins zum Thema zu machen. ${ }^{12}$ Scheint Dekonstruktion doch in keiner Weise dem Motiv des historischen Gedächtnisses, sondern allenfalls der kritischen Auflösung des Vergangenen verpflichtet. Indessen zeigt sich bei näherem Hinsehen, dass solche

\footnotetext{
${ }^{10}$ Foucault, « Nietzsche, la généalogie, l'histoire », S. 154 (dt. S. 106).

${ }^{11}$ Vgl. Michel Foucault, L'ordre du discours, S. 62f, S. 67f.

${ }^{12}$ Zum Folgenden ausführlicher: VF., Interpretation und Dekonstruktion. Untersuchungen zur Hermeneutik, Kap. 11 und 12, Weilerswist, Velbrück Wissenschaft, 2003.
} 
Einschätzung nur einen Oberflächenaspekt der dekonstruktiven Praxis reflektiert. In Wahrheit lässt sich gerade von der Dekonstruktion zeigen, dass sie in einem genuinen Sinn der Geschichte verpflichtet, « von Fall zu Fall durch eine Sorge um die Geschichte motiviert ${ }^{13}$ ist. In unserem Zusammenhang von besonderem Interesse ist sie dadurch, dass sie unterschiedliche Einstellungen des historischen Bewusstseins verkörpert, die sich in gewisser Analogie zu den Typen der Historie bei Nietzsche und zu deren Spezifizierung bei Foucault setzen lassen. Dekonstruktion ist der Titel für eine bestimmte Weise des Umgehens mit Texten, Traditionen und Sinngebilden, die deren überlieferte Gestalt aufbricht, um sie zugleich weiterzuentwickeln, zu hinterfragen und neu zu formulieren. Sie ist eine Weise, an Geschichte teilzunehmen, die drei verschiedene Stoßrichtungen vereinigt: die Destruktion, die Konstruktion und die Rezeption. Diese drei Richtungen sind kurz für sich zu kennzeichnen und dann im Horizont des Geschichtsdenkens zu situieren.

Ihrem Namen nach ist Dekonstruktion vor allem eine Verbindung der beiden ersten, Destruktion und Konstruktion, Auflösung und Neubildung; als ihr distinktives Merkmal erscheint zunächst das erste, die kritische Auflösung. Sie will die Gestalt, in der uns Traditionsbestände gegeben sind, auflösen, ihre Ansprüche hinterfragen, ihre vermeintliche Eindeutigkeit problematisieren. Was eine historische Situation bedeutet, was ein Text aussagt, wozu eine Institution bestimmt ist - all dies erschließt sich nicht dem unmittelbaren Blick, lässt sich nicht der proklamierten Selbstdefinition entnehmen. Der Gegenstand ist nicht nur in seiner manifesten Gestalt, sondern auch in seiner Latenz und Möglichkeitsdimension, in dem, was er verbirgt, zu begreifen; der Inhalt eines Textes wird nicht nur durch das « in ihm » Gesagte, sondern ebenso durch den äußeren Kontext, aus dem er kommt und mit dem er kommuniziert, bestimmt. Zuweilen macht gerade das Nichtgesagte, das Nichtthematische und an den Rand Gedrängte die wahre Bedeutung eines Textes, einer Praktik, einer Lebensgeschichte aus. Die dekonstruktive Analyse kann die unterschiedlichsten Modi der Vermittlung von Innen und Außen, der Verschränkung von Text, Intertext und Kontext in Rechnung stellen. Unübersehbar ist die Nähe zu den Operationen, die Foucault als Momente der genealogischen Kritik beschreibt, aber auch zu Motiven einer kritischen Historie oder einer kritischen Hermeneutik, wie sie beispielsweise Ricœur unter dem Stichwort einer Hermeneutik des Verdachts im Anschluss an Nietzsche, Freud und Marx zeichnet. Hier geht es darum, Selbstmissdeutungen zu kritisieren,

13 Jacques DERrIDA, Einige Statements und Binsenweisheiten über Neologismen, New-Ismen, Post-Ismen, Parasitismen und andere kleine Seismen, Berlin, Merve, 1997, S. 59. 
Sinnverzerrungen in ihrer Genese und ihrer Funktion aufzudecken, die verfestigte Gestalt einer Deutungsgeschichte aufzubrechen und neu zu konstellieren. Weit über pathologische Befunde hinaus bringt Dekonstruktion die hermeneutische Einsicht zur Geltung, dass zwischen dem Subjekt und seinem bewussten Meinen, aber auch zwischen seinem Meinen (Sagenwollen) und seinem faktischen Sprechen (Ausdrücken) eine Kluft herrscht, die zur interpretierenden Reformulierung nötigt, dass die Aussage jedes Sprechenden immer «mehr, weniger oder anderes sagt, als was er sagen wollte ${ }^{14}$. Erst recht ist diese Kluft Anlass der kritischen Auflösung dort, wo sie in der Produktion von falschem Bewusstsein und realen Verzerrungen resultiert.

Nun ist die Dekonstruktion, indem sie sowohl die Tiefenschicht im Text wie den äußeren Kontextbezug zur Geltung bringt, nicht einfach Auflösung des Vorgegebenen, sondern ebenso Konstruktion und Neubildung. Das Auflösen ist Kehrseite eines Neuschreibens der Geschichte. Im Fall der Lektüre und Neuschreibung von Texten spezifiziert Derrida diese Operation als Umkehrung und Neukonstellierung der Gegensätze, die das semantische Feld und seine logische Struktur bestimmen: der Gegensätze von Zentrum und Peripherie, Wesentlichem und Unwesentlichem, Sinn und Genese. Dekonstruktion bringt in den Blick, was im Text verborgen, marginalisiert war; sie betreibt die ReThematisierung dessen, was von der Macht der Überlieferung zum Impliziten reduziert, ausgeschlossen und verdrängt wurde. Für die Geschichte liegt darin eine Neubestimmung des Verhältnisses zwischen Interpretation und Gegenstand. Dekonstruktion macht sich selbst zum Teil des Sinnbildungsprozesses, den sie, indem sie ihn zu ergründen und auszulegen sucht, mitkonstituiert und neu gestaltet. Geschichte und dekonstruktive Lektüre durchdringen sich gegenseitig: Im Prozess der Dekonstruktion ist die Geschichte am Werk, wie umgekehrt in der Geschichte als reflexivem Prozess die dekonstruktive Tätigkeit wirksam ist. Dekonstruktion trifft sich darin mit einem von der Hermeneutik emphatisch herausgestellten Zug des Verstehens: Gadamer wie Ricoeur unterstreichen die «Zugehörigkeit» des Verstehens zum geschichtlichen Sinngeschehen, in welchem das Verstehen seinen Grund und seine Grenze hat. In der Dialektik von vorgängiger Zugehörigkeit und kreativem Hervorbringen ist auch ein Gegenakzent gegen eine primär konstruktivistische Sichtweise gesetzt, wie sie in neueren Strömungen des "Interpretationismus », u.a. im Anschluss an Nietzsche, betont worden ist. Historische Konstitution ist gerade nicht reine Konstruktion.

\footnotetext{
${ }^{14}$ Jacques DERRIDA, De la grammatologie, Paris, Minuit, 1967, S. 226.
} 
Die Affinität zum Geschichtlichen erhält eine neue Tiefendimension, wenn neben den Momenten des Destruierens und Konstruierens das Moment des Aufnehmens und Bewahrens in den Blick kommt. Es ist das Moment, das dem dekonstruierenden Umgang mit Texten zunächst am meisten fremd zu sein scheint. In Wahrheit jedoch ist Dekonstruktion in durchaus prägnanter Weise durch den Bezug auf die Überlieferung definiert. Nicht nur ist sie, wie im Vorigen betont, Teil des geschichtlichen Prozesses; ebenso ist sie thematisch auf Geschichte bezogen, ist sie wesentlich über den Bezug auf dasjenige, an das sie anschließt und das sie weiterschreibt, definiert. $\mathrm{Zu}$ ihren Eigentümlichkeiten gehört die Anlehnung an vorgegebene Traditionsbestände: Dekonstruktives Philosophieren realisiert sich bevorzugterweise in der Abarbeitung an überlieferten Texten und Problemstellungen. Dies aber heißt: Es verbindet die destruierend-konstruierende Arbeit am Gegenstand mit dem Interesse an dessen Erschließung, am Verstehen dessen, was ein Text sagt und was eine Tradition bedeutet. Zwar will sich Dekonstruktion dezidiert von den Leitideen traditionellen Geschichtsdenkens, etwa der Unterstellung eines identischen Sinns, der sich in der Geschichte teleologisch entfaltet, distanzieren. Doch bedeutet dies keine Abschwächung des geschichtlichen Zusammenhangs. Wenn Dekonstruktion die Brüche und Neuerungen hervorhebt, so betont sie mit gleichem Gewicht die Rückbindung an das Dekonstruierte: Jeder neue Entwurf enthält alle früheren in sich, indem er « sie in sich einschreibt. Jeder Entwurf wird strukturiert, konstruiert, entworfen, um von allen anderen Entwürfen (vergangenen, gegenwärtigen und sogar zukünftigen) Rechenschaft abzulegen und aufzuzeigen, worin sie gründen $»^{15}$. Dekonstruktives Philosophieren ist ein Schreiben, das in einem Lesen gründet, das hinter das explizit Gesagte zurückgehen will. Auch wenn es darin zu keinem absolut Ersten - wie umgekehrt das Schreiben zu keinem abschließenden Sagen des Gemeinten kommt, steht dekonstruierendes Anknüpfen im Dienste der Erschließung des Gesagten (und Nicht-Gesagten), der Artikulation des Sinnes, der unterwegs ist.

Exemplarisch bringt Derrida die Aufgabe solcher Neuschreibung in der Figur der Übersetzung zur Sprache, wie er sie in W. Benjamins Aufsatz über " Die Aufgabe des Übersetzers » artikuliert sieht. ${ }^{16}$ Übersetzung ist dabei nicht als bloße Übertragung eines Inhalts von einem Idiom in ein anderes, sondern im weiten Sinn als Interpretation verstanden, als ein Zur-Sprache-Bringen dessen, was in einem Text gemeint, doch noch unzulänglich ausgedrückt - oder gerade

\footnotetext{
${ }^{15}$ DERrIDA, Einige Statements, S. 9.

${ }^{16}$ Jacques DERRIDA, " Des tours de Babel », in: Psyché. Inventions de l'autre, Paris, Galilée, 1987, S. 203-235.
} 
verhüllt - ist. Derrida verallgemeinert die Figur nach beiden Seiten: Dem immer unvollständigen, defizienten Ausdruck, der nach seiner Ergänzung verlangt, entspricht eine ursprüngliche Pflicht des Deutens, das dem Gemeinten und zu Sagenden gerecht zu werden hat. Zur Aufgabe wird Dekonstruktion mit Rücksicht auf das, was im Text gesagt ist und gleichwohl zu sagen bleibt, auf das Gedachte, das « noch gedacht werden muss ${ }^{17}$. Dekonstruktion verkörpert eine profilierte Version eines Geschichtsdenkens, das einen radikalen Zukunftsbezug in einem vertieften Vergangenheitsbezug begründet. Es ist eine Begründung, die gegen das teleologische Entwicklungsmodell klassischer Geschichtsphilosophie die direkte Gegenfigur zeichnet. Nicht was der Ursprung ist und was er als Keim enthält, sondern was fehlt und unterdrückt ist, ist der Impuls historischen Gedenkens. Wenn Derrida davon spricht, dass Dekonstruktion im « Sinn für eine grenzenlose [...] Verantwortung gegenüber dem Gedächtnis » und der Geschichte gründet ${ }^{18}$, so gilt auch dies in einem Benjamin verwandten Sinn: Nicht als Verantwortung gegenüber dem faktischen Verlauf, sondern als Widerstand gegen den bloßen Machtspruch der Geschichte und als Rettung dessen, was in ihr unterdrückt und unerfüllt geblieben ist.

Dekonstruktion erweist sich so als eine Partizipation am Sinngeschehen, die in pointierter Form drei unterschiedliche Grundhaltungen - Destruktion, Konstruktion, Bewahrung - gegenüber der Geschichte vereinigt, in denen wir - neben Verschiebungen - einen Anklang an Nietzsches drei Potenzen der Historie erkennen können. Wenn bei Nietzsche offenblieb, wie die drei Figuren in eine historische Praxis zu integrieren sind, so enthält die Dekonstruktion die These ihrer notwendigen Komplementarität. Es ist eine Praxis der Aneignung von Geschichte, die in sich verschiedene Strukturmomente in einem spannungsvollen Wechselspiel zusammenhält.

\section{Geschichtlichkeit und Konstruktion}

Die Divergenz historischer Orientierungen, die wir bei Nietzsche erkennen, ist in der Folgegeschichte noch deutlicher hervorgetreten, teils in der zyklischen

\footnotetext{
${ }^{17}$ Jacques DERRIDA, L'autre cap, Paris, 1991, 75 f.; vgl. ID., Limited Inc., Paris, Galilée, 1990, S. 237.

${ }^{18}$ Jacques Derrida, Force de loi. Le "Fondement mystique de l'autorité ", Paris, Galilée, 1994, S. 44 (dt.: Gesetzeskraft. Der « mystische Grund der Autorität », Frankfurt/Main, Suhrkamp, 1991, S. 40).
} 
Ablösung von Historisierungs- und Enthistorisierungsbewegungen, teils innerhalb der einzelnen Konzepte und Dispositive historischer Arbeit. Wichtig scheint dabei zunächst die Feststellung, dass die Zurückweisung übergreifender Einheits-, Fortschritts- und Vernunftpostulate, vom Historismus bis zur Postmoderne, keineswegs mit einer Verabschiedung historischen Denkens einhergehen muss, sondern sich durchaus in dessen Dienst stellen kann. Die Anerkennung von Pluralität und Kontingenz ist konstitutiv für geschichtliches Denken. In diesem Sinn finden wir bei Autoren wie Foucault und Derrida, die mit Nachdruck die Zersetzung aller geschichtsphilosophischen Hypostasierungen fordern, zugleich ein leidenschaftliches Bemühen um Geschichte und historische Erkenntnis. Ihre historisch-genealogischen Rekonstruktionen treten mit dem unleugbaren Anspruch einer sachhaltigeren, radikaleren, " wahreren » Vergegenwärtigung der Geschichte auf. Dabei sind der kritisch-auflösende, der bewahrend-erschließende und der konstruktivinterpretierende Aspekt in ihrer Arbeit weder gegeneinander auszuspielen noch voneinander abzulösen. Als Fazit können wir gerade im Rückblick auf ihre historische Arbeit die nicht-reduzierbare Heterogenität divergierender Ansätze historischer Erkenntnis festhalten. Wir brauchen alle, ohne sie aufeinander zurückführen zu können.

Ein analoger Schluss drängt sich im Blick auf die Frage nach dem grundsätzlichen Bezug zur Geschichte bzw. dem Verhältnis zwischen historischer und unhistorischer (oder antihistorischer) Orientierung auf. Geschichte ist uns problematisch und unhintergehbar zugleich. Dabei geht es nicht nur um eine Ambivalenz erkenntnisleitender oder existentieller Interessen. Im Spiel ist ein struktureller Antagonismus zwischen der Konstruktivität und der Historizität unseres Selbst- und Weltverhältnisses. Zahlreiche Autoren haben in den letzten Jahrzehnten den konstruktiven Anteil (die narrative Konstitution, die Erinnerungsarbeit, die temporale Synthesis) in unserer Wahrnehmung der Geschichte unterstrichen; andere haben darüber hinaus die interpretativ-kreative Dimension unseres Wirklichkeitsverhältnisses im Ganzen herausgearbeitet. Menschen sind, was sie sind, kraft ihrer Selbstinterpretation und Selbsthervorbringung; ihr Sein geht weder in einer Wesensbestimmung noch einem historischen Sich-Gegebensein auf, sondern schließt den Entwurf, das Experiment, die Selbsterschaffung ein. Zum Teil enthält auch ihr historisches Sein eine Seite dieses Experimentierens, wie Nietzsche und Foucault mit Bezug auf den historischen Karneval der Masken illustrieren. Indessen ist nicht zu übersehen, dass gerade die Geschichte auch die Grenze jeder Selbsterzeugung und Selbstvariierung markiert. Im historischen Bewusstsein wird der Mensch 
nicht nur des eigenen Möglichkeitsraums, sondern ebenso der unhintergehbaren Faktizität seines Seins und Soseins gewahr: Zum historischen Erkennen gehört auch die Anerkenntnis des vorgängigen Sich-Gegebenseins. Historische Identitätsbildung, auch wenn sie nur über das eigene Verstehen und konstruktive Auslegen der Geschichte zustandekommt, geht nicht im subjektiven Akt auf. So verbleibt, noch grundlegender als die Divergenz der Formen historischer Arbeit, die unauflösbare Spannung zwischen der Geschichtlichkeit und der Kreativität des menschlichen Seins. Die Geschichte, ein menschlich-kulturelles Konstrukt, ist nach anderer Hinsicht das radikal Nichtkonstruierbare. Menschliches Sein ist zwischen Konstruktion und dem Nichtkonstruierbaren ausgespannt. Dass wir geschichtliche Wesen sind, macht unsere menschliche Verfassung aus, die uns ermöglicht und uns dazu auffordert, Entwürfe und Beschreibungen unserer selbst hervorzubringen, die uns darin aber auch mit der unverrückbaren Grenze jeder Selbsterschaffung konfrontiert. Dies scheint der tiefste Zwiespalt, zu dem uns die mehrfachen Ambivalenzen des Historischen zurückführen. 